\title{
AN UNCONDITIONALLY STABLE SPLITTING SCHEME FOR A CLASS OF NONLINEAR PARABOLIC EQUATIONS
}

\author{
KENNETH HVISTENDAHL KARLSEN AND KNUT-ANDREAS LIE
}

\begin{abstract}
We propose and analyse a numerical scheme for a class of advection dominated advection-diffusion-reaction equations. The scheme is essentially based on combining a front tracking method for conservation laws, which tracks shock curves defined by a varying velocity field, with a suitable operator splitting. The splitting is formulated for an equation in non-conservative form and consists of a nonlinear conservation law modelling advection, a heat equation modelling diffusion, and finally an ordinary differential equation modelling lower order processes. Since no CFL condition is associated with the front tracking scheme, our numerical scheme is unconditionally stable in the sense that the splitting time step is not restricted by the spatial discretization parameter. Nevertheless, it is observed that when the splitting time step is notably larger than the diffusion scale, the scheme can become too diffusive. This can be inferred with the fact that the entropy condition forces the hyperbolic solver to throw away information regarding the structure of shock fronts. We will demonstrate that it is possible to identify what is thrown away as a residual flux term. Moreover, if this residual flux is taken into account via, for example, a separate correction step, the shock fronts can be given the correct amount of self sharpening. Two numerical examples are presented and discussed. The first is an academic test case while the second is drawn from glacier modelling.
\end{abstract}

\section{INTRODUCTION}

Mathematical models that involve a combination of advective, diffusive, and reactive processes are among the most widespread in all of sciences, engineering, and other fields where mathematical modelling is important. Applications range from models of turbulence [4], via traffic flow [35] and modelling of share options on the financial market [3], to multiphase flow in porous media [42]. Other applications include, to mention a few, polymer chemistry [6], combustion modelling [37], modelling of semi-conductor devices [36], and contaminant transport in groundwater and surface water [2].

We consider a class of nonlinear advection-diffusion-reaction equations of the form

$$
\frac{\partial}{\partial t} u+\frac{\partial}{\partial x} F(x, t, u)=\varepsilon \frac{\partial^{2}}{\partial x^{2}} u+G(x, t, u), \quad(x, t) \in Q_{T} \equiv \mathbb{R} \times\langle 0, T],
$$

Key words and phrases. Nonlinear advection-diffusion-reaction equation, operator splitting, front tracking.

The research of the first author has been supported by VISTA, a research cooperation between the Norwegian Academy of Science and Letters and Den norske stats oljeselskap a.s. (Statoil). The research of the last author has been supported by the Research Council of Norway under grant 100555/410. 
with initial data $u_{0}$ imposed at $t=0$. Here the flux function $F$ and the reaction $/$ source/sink term $G$ are bounded, smooth functions of all their arguments; and the initial data $u_{0}$ is of bounded total variation.

When (1) is advection dominated, conventional numerical methods exhibit some combination of difficulties, from non-physical oscillations (central difference/Galerkin schemes) to excessive numerical diffusion (upwind schemes) at the trailing end of moving shock fronts. In the last two decades we have seen a tremendous activity on developing sophisticated numerical schemes (e.g., finite difference, finite element, finite volume, Godunov, and front tracking methods) that are designed to accurately capture discontinuous solutions of hyperbolic conservation laws $[22,27,33]$. It may therefore seem reasonable to employ some of these methods as "building blocks" in numerical schemes for advection-diffusion-reaction problems, at least for those that are of advection dominated nature.

A natural strategy is to split the equation into a hyperbolic conservation law modelling advection, a parabolic equation modelling diffusion, and finally an ordinary differential equation modelling "lower order terms", and then try to reproduce the solution using these simpler equations as building blocks. Variations on this operator splitting approach have indeed been taken several authors, we mention Demkowitz and Oden [15], Douglas and Russell [16], Espedal and Ewing [17], Dahle [11], Arbogast and Wheeler [1]. A more general approach is the Eulerian-Lagrangian localized adjoint methods of Celia et al. [5] and Wang et al. [44]. We also refer to [14, 19, 20, 23, 40, 41, 47], and the references therein, for more details on this type of methods, which are all based on some kind of time stepping along the characteristics to realize the hyperbolic part of (1). Dawson [12, 13, 48] employs Godunov/upwind methods to realize the hyperbolic part of (1), giving an approach closer to the one we advocate here. We also mention the recent work of Cockburn and Shu [7] on the (unsplit) local discontinuous Galerkin method, which is an extension of the RungeKutta Discontinuous Galerkin method for hyperbolic conservation laws.

The purpose of our paper is to continue and improve the work initiated recently by Karlsen, Risebro, Lie, and coworkers on the corrected operator splitting (COS) approach $[31,30,28,29]$. This approach is based on a front tracking method for conservation laws and a dynamical splitting of the partial differential equation (1) in the case $F \equiv f(u), G \equiv 0$. An ultimate goal is to accurately and effectively solve the partial differential equations describing multi-phase flow in porous media, including the strongly heterogeneous case where the coefficients in (1) vary discontinuously with the space variable. Although we will use a simple linear diffusion term $\varepsilon u_{x x}$ here, a nonlinear and possibly strongly degenerate diffusion term $\varepsilon\left(d(x, t, u) u_{x}\right)_{x}$ could have been considered. But in the degenerate case, weak solutions must be sought and they are, in general, not uniquely determined by their data. Consequently, an entropy condition must be imposed to pick out the physical weak solution. The use of entropy conditions (inequalities) are outside the scope of this paper, and we refer instead to Evje and Karlsen [18] for details in the case $F \equiv f(u)$ and $G \equiv 0$.

We restrict ourselves to a (physically reasonable) class of equations in which the flux function $F$ has the form $F(x, t, u)=V(x, t) f(u)$. This will enable us to write the parabolic 
equation (1) in the non-conservative form

$$
\frac{\partial}{\partial t} u+V(x, t) \frac{\partial}{\partial x} f(u)=\varepsilon \frac{\partial^{2}}{\partial x^{2}} u+Q(x, t, u),
$$

where $Q(x, t, u)=G(x, t, u)-f(u) V_{x}(x, t)$. This equation will be our starting point for constructing approximate solutions. Let $\mathcal{S}(t), \mathcal{H}(t)$, and $\mathcal{R}(t)$ denote the solution operators associated with the hyperbolic equation $v_{t}+V(x, t) f(v)_{x}=0$, the parabolic equation $w_{t}=\varepsilon w_{x x}$, and the ordinary differential equation $z_{t}=Q(x, t, z)$, respectively. Then the basic splitting takes the form

$$
u(x, n \Delta t) \approx[\mathcal{R}(\Delta t) \circ \mathcal{H}(\Delta t) \circ \mathcal{S}(\Delta t)]^{n} u_{0} .
$$

Later we will replace $\mathcal{S}(t)$ with a front tracking method, $\mathcal{H}(t)$ with an explicit difference scheme, and $\mathcal{R}(t)$ with the forward-Euler method. Note, that the main emphasis in this paper will be on front tracking and the corrected operator splitting. Consequently, our choice of numerical schemes for $\mathcal{H}(t)$ and $\mathcal{R}(t)$ will be very simple to allow us to focus on the main ideas of our solution strategy as well as keeping the details in the convergence analysis at a minimum.

The front tracking method we will use was first introduced by Dafermos [10], then suggested as a numerical method by Holden, Holden, and Høegh-Krohn [25], and later extended to systems by Risebro [39]. Our method deviates slightly from the original [25] in that we track shock curves in a piecewise linear velocity field, yielding curves that vary piecewise exponentially in time as opposed to piecewise linearly. Applying front tracking for the advective step has some striking advantages. First, there is no CFL condition associated with this method. Hence the resulting splitting scheme is unconditionally stable in the sense that the time step $\Delta t$ is not restricted by the spatial discretization parameter. Second, as we shall see later, the method easily allows for a dynamical splitting of the equation (2), which contrasts the fixed splitting (3) that is usually employed [12, 13, 31]. Although unconditionally stable, the method contains splitting errors. This means that a reasonable choice of $\Delta t$ is highly dictated by the degree of interplay between advective, diffusive, and reactive forces. Here we will focus on the interaction between advection and diffusion. A thorough discussion of the influence from reactive terms is left to a separate report. Instead, we refer to some papers $[9,26,32,45,46]$ concerned with splitting off lower order terms in hyperbolic and parabolic conservation laws.

Let us for the moment assume that $Q \equiv 0$ so that (2) is of advection-diffusion type. When $\Delta t$ is larger than the diffusion scale $\varepsilon$, the splitting (3) is too diffusive near selfsharpening shock fronts [28,31]. This happens because the entropy condition forces the hyperbolic solver $\mathcal{S}(\Delta t)$ to throw away the information controlling the structure of the (self-sharpening) fronts. We will show how to compensate for this "loss of information", which manifests itself in the form of a residual flux term $f_{\text {res }}$. For instance, assume that the solution of (2) is simply a moving shock front. Applying $\mathcal{S}(\Delta t)$ gives a discontinuity, with left and right limits $u_{l}$ and $u_{r}$, respectively. Then we can identify the residual term as $f_{\text {res }}=f-f_{c}$, where $f_{c}$ denotes the correct envelope (dictated by the entropy condition) of $f$ in the interval bounded by $u_{l}$ and $u_{r}$. There are several ways to take the residual 
flux term $f_{\text {res }}$ into account. We can, for instance, perform a separate correction step after the diffusion step. Correction is then realized by solving the "residual" conservation law $v_{t}+V(x, t) f_{\text {res }}(v)_{x}=0$ over a time interval $\langle 0, \tau]$, where $\tau>0$ is some parameter that has to be chosen. Another approach is to include the residual term in the equation modelling diffusion, that is, instead of solving (only) the heat equation $w_{t}=\varepsilon w_{x x}$, we solve $w_{t}+V(x, t) f_{\text {res }}(v)_{x}=\varepsilon w_{x x}$. The point is that this equation contains the necessary information needed to produce the correct structure of the shock front, but contains a less severe nonlinearity than (2). We will later discuss and apply both approaches.

A simpler form of the techniques developed here has been implemented in a two-dimensional prototype black oil simulator [29]. The idea is to treat multi-dimensional equations by means of dimensional splitting. To be more precise, let us consider the following multidimensional analogue of (1)

$$
\frac{\partial}{\partial t} u+\sum_{i=1}^{d} \frac{\partial}{\partial x_{i}} F_{i}\left(x_{1}, \ldots, x_{d}, t, u\right)=\varepsilon \sum_{i=1}^{d} \frac{\partial^{2}}{\partial x_{i}^{2}} u+G\left(x_{1}, \ldots, x_{d}, t, u\right) .
$$

Letting $\mathcal{E}_{\Delta}^{x}(t)$ denote our approximate one-dimensional solution (evolution) operator associated with (1), we can then realize the solution of (4) using the product formula

$$
u\left(x_{1}, \ldots, x_{d}, n \Delta t\right) \approx\left[\mathcal{E}_{\Delta}^{x_{d}}(\Delta t) \circ \cdots \circ \mathcal{E}_{\Delta}^{x_{1}}(\Delta t)\right]^{n} u_{0} .
$$

For scalar hyperbolic equations, combining front tracking and dimensional splitting gives not only a simple, but also an efficient and reliable method [34]. This approach is also attractive for $(5)$ since each $\mathcal{E}_{\Delta}^{x_{i}}(t)$ represents an unconditionally stable scheme, which implies that a large time step $\Delta t$ is feasible. In [29], a Petrov-Galerkin finite element method is employed to solve the diffusion equation instead of the simple finite difference scheme used here. Compared with a standard approach, the results are very promising. By combining the analysis found in $\S 3$ with the analysis found in [31], it is possible to prove convergence of the multi-dimensional splitting formula (5); we omit the details.

Our paper is organized as follows. In $\S 2$ we describe in detail the front tracking method and the construction of the (corrected) operator splitting solutions. In $\S 3$ we establish convergence of these approximate solutions by making use of a $L_{1}$ compactness argument. Finally, in $\S 4$ we present and discuss some numerical applications of our solution strategy.

\section{Construction of Approximate Solutions}

The splitting approach results in three different equations that each must be solved numerically. Therefore, before we describe the operator splitting in more detail, we introduce numerical schemes for solving these three equations. But as mentioned earlier, the schemes for the diffusive and the reactive part are deliberately chosen as simple as possible in order to focus on the main ideas of our front tracking/corrected splitting strategy. For less academic purposes, these simple methods obviously can and will be replaced by other schemes. 
Advection Equation. Let us begin with front tracking. Consider therefore the nonlinear conservation law

$$
\frac{\partial}{\partial t} v+V(x, t) \frac{\partial}{\partial x} f(v)=0, \quad v(x, 0)=v_{0}(x)
$$

where we assume that $f(v)$ is a continuous piecewise linear function and $v_{0}(x)$ is a step function with a finite number of jumps. The piecewise constant initial function yields a sequence of Riemann problems of the form

$$
v(x, 0)= \begin{cases}v_{l}, & \text { when } x<0 \\ v_{r}, & \text { when } x>0\end{cases}
$$

Each of these Riemann problems can be solved analytically, and since the flux function $f$ is piecewise linear, the solution will be a step function. Rarefaction waves are replaced by sequences of small shocks. Recall that a discontinuity $\left(v_{l}, v_{r}\right)$ with associated shock speed $s=\left(f\left(v_{l}\right)-f\left(v_{r}\right)\right) /\left(v_{l}-v_{r}\right)$ is admissible provided the entropy condition

$$
\operatorname{sgn}(V(x, t))\left(\frac{f(v)-f\left(v_{r}\right)}{v-v_{r}}\right) \leq \operatorname{sgn}(V(x, t)) s \leq \operatorname{sgn}(V(x, t))\left(\frac{f(v)-f\left(v_{l}\right)}{v-v_{l}}\right)
$$

is satisfied for all $v$ between $v_{l}$ and $v_{r}$. This condition, which is due to Oleinik [38], ensures that the solution is physically correct. To give the complete solution of the Riemann problem, assume first that $V(x, t)$ is positive and introduce the envelope function

$$
f_{c}\left(v ; v_{l}, v_{r}, V\right)=\left\{\begin{array}{l}
\text { the lower convex envelope of } f \text { between } v_{l} \text { and } v_{r} \text { if } v_{l}<v_{r} \\
\text { the upper concave envelope of } f \text { between } v_{r} \text { and } v_{l} \text { if } v_{l}>v_{r} .
\end{array}\right.
$$

For negative $V(x, t)$ the envelope function $f_{c}$ is defined similarly with the roles of convex and concave interchanged. Suppose that $\left\{v_{i}\right\}$ are the breakpoints of $f$, i.e., the points where $f^{\prime}$ is discontinuous. Since $f$ is piecewise linear, then so is $f_{c}$, and the breakpoints $\left\{\bar{v}_{i}\right\}$ of $f_{c}$ form a subset of $\left\{v_{i}\right\}$. If we set $\bar{v}_{1}=v_{l}$ and $\bar{v}_{M}=v_{r}$, we can write the solution of the Riemann problem as

$$
v(x, t)= \begin{cases}v_{l}, & x<\bar{x}_{0}(t) \\ \bar{v}_{i}, & \bar{x}_{i}(t) \leq x \leq \bar{x}_{i+1}(t), \quad i=1, \ldots, M-1, \\ v_{r}, & x>\bar{x}_{M-1}(t),\end{cases}
$$

where each $\bar{x}_{i}(t)$ satisfies a Rankine-Hugoniot condition

$$
\frac{d \bar{x}_{i}}{d t}=V\left(\bar{x}_{i}, t\right)\left(\frac{\bar{f}_{i+1}-\bar{f}_{i}}{\bar{v}_{i+1}-\bar{v}_{i}}\right)=V\left(\bar{x}_{i}, t\right) \bar{s}_{i},
$$

with $\bar{f}_{i}=f_{c}\left(\bar{v}_{i} ; v_{l}, v_{r}\right)$. The shock paths $\bar{x}_{i}(t)$ can be computed explicitly if the function $V(x, t)$ has a simple form. For instance, if $V=V(x)=(a x+b)$, the path of a shock with speed $s$ starting from $x_{0}$ at time $t_{0}$ is given by $x(t)=\left(x_{0}+b / a\right) \exp \left[a s\left(t-t_{0}\right)\right]-b / a$, where $a$ is nonzero. Connecting the solutions of the local Riemann problems gives the global solution of (6) up to the first time when two waves from neighbouring Riemann problems interact. This interaction defines a new Riemann problem with left and right states given 
by the values immediately to the left and right of the collision point. The new Riemann problem is then solved as outlined above, thereby, giving the global solution until the next interaction occurs, and so on. Summing up, the front tracking algorithm goes as follows:

- Solve the Riemann problems defined by the piecewise constant initial data.

- Keep track of shock collisions and solve Riemann problems at the collision points.

However, note that special care has to be taken when the sign of the velocity changes with time. Since the entropy condition is dependent upon $\operatorname{sgn}(V(x, t))$, all Riemann problems then have to be solved again if we wish to advance correctly past a point $\tau$ for which $V(x, \tau)=0$. The front tracking method for a general conservation law (arbitrary $V, f$, and $v_{0}$ ) consists in replacing $f$ with a piecewise linear approximation, $v_{0}$ with a piecewise constant approximation, and $V$ by a function on a form such that (11) can be solved explicitly; typically piecewise linear in space and time, which is assumed from now on. Then an approximate solution is found by solving the resulting (perturbed) problem exactly according to the procedure described above. For a more detailed treatment of the front tracking method in the constant coefficient case we refer to [25].

Diffusion Equation. As pointed out earlier, diffusion is modelled by realizing an equation of the form

$$
\frac{\partial}{\partial t} w=\varepsilon \frac{\partial^{2}}{\partial x^{2}} w, \quad w(x, 0)=w_{0}(x)
$$

where we assume that the data $w_{0}(x)$ is of bounded variation. When integrating a parabolic equation like (12), one can choose from a diversity of numerical schemes. We will here simply employ an explicit finite difference scheme. Let $h$ and $k$ be the spatial and temporal increments, that is, $x_{i}=i h$ and $t_{m}=m k$ for $i \in \mathbb{Z}$ and $m \in \mathbb{Z}_{+}$. We assume that the stability condition $\alpha \equiv \varepsilon k / h^{2} \leq 1 / 2$ is satisfied. The approximation to $w\left(x_{i}, t_{m}\right)$ will be denoted by $w_{i}^{m}$. To start the scheme we set $w_{i}^{0}=\left(\pi w_{0}\right)\left(x_{i}\right)$, where $\pi$ denotes the usual grid cell averaging operator on $\left\{x_{i} ; h\right\}$. We compute $w_{i}^{m+1}$ from the finite difference equation

$$
w_{i}^{m+1}=\alpha w_{i-1}^{m}+(1-2 \alpha) w_{i}^{m}+\alpha w_{i+1}^{m}, \quad(i, n) \in \mathbb{Z} \times \mathbb{Z}_{+} .
$$

For later use, introduce the interpolation $w_{\Delta}(x, t)=w_{i}^{m}$ for $(x, t) \in\left[x_{i}, x_{i+1}\right\rangle \times\left[t_{m}, t_{m+1}\right\rangle$.

Reaction Equation. To realize reactive/source/sink processes we use the forward-Euler method to solve an ordinary differential equation of the form

$$
\frac{\partial}{\partial t} z=Q(x, t, z), \quad z(x, 0)=z_{0}(x)
$$

To start the scheme set $z_{i}^{0}=z_{0}\left(x_{i}\right)$. We compute $z_{i}^{m+1}$ from $z_{i}^{m}$ using the equation

$$
z_{i}^{m+1}=z_{i}^{m}+k Q_{i}^{m}, \quad(i, n) \in \mathbb{Z} \times \mathbb{Z}_{+}, \quad Q_{i}^{m}=Q\left(x_{i}, t_{m}, z_{i}^{m}\right) .
$$

For later use, introduce the interpolation $z_{\Delta}(x, t)=z_{i}^{m}$ for $(x, t) \in\left[x_{i}, x_{i+1}\right\rangle \times\left[t_{m}, t_{m+1}\right\rangle$. 
Splitting Algorithm. From now on, let $h>0$ and $\Delta t>0$ denote the spatial and temporal discretization parameters, i.e., $x_{i}=i h$ for $i \in \mathbb{Z}$ and $t_{n}=n \Delta t$ where $n \leq N$ and $N \Delta t=T$. Define $V^{\Delta}(x, t)$ and $f^{\delta}(u)$ to be proper piecewise linear approximations to $V(x, t)$ and $f(u)$, respectively $(\Delta$ and $\delta>0$ denote polygonal approximation parameters). Assume that we know the approximate solution $u^{n}$ at time $t=t_{n}$, for some fixed $n<N$. Next, we describe how to construct $u^{n+1}$ from $u^{n}$.

Step 1 (advection): Let $v(x, t)=\mathcal{S}_{\Delta}(t) u^{n}$ be the front tracking solution to the conservation law

$$
\frac{\partial}{\partial t} v+V^{\Delta}(x, t) \frac{\partial}{\partial x} f^{\delta}(v)=0, \quad v(x, 0)=u^{n}(x), \quad(x, t) \in \mathbb{R} \times\langle 0, \Delta t],
$$

and introduce the intermediate solution $u^{n+1 / 3}=\mathcal{S}_{\Delta}(\Delta t) u^{n}$.

Step 2 (diffusion): Choose a local time step $k_{d}$ such that $k_{d} N_{d}=\Delta t$ and $\alpha_{d}=\varepsilon k_{d} / h^{2} \leq$ $1 / 2$. Let $w_{\Delta}(x, t)=\mathcal{H}_{\Delta}(t) u^{n+1 / 3}$ be the finite difference solution of the parabolic problem

$$
\frac{\partial}{\partial t} w=\varepsilon \frac{\partial^{2}}{\partial x^{2}} w, \quad w(x, 0)=u^{n+1 / 3}(x), \quad(x, t) \in \mathbb{R} \times\langle 0, \Delta t] .
$$

Then introduce the intermediate solution $u_{-}^{n+2 / 3}=\mathcal{H}_{\Delta}(\Delta t) u^{n+1 / 3}$.

As pointed out earlier, it can be necessary to do a correction step to get the structure of the shock fronts right. To this end, recall that the function $u^{n+1 / 3}(x)$ is piecewise constant on a finite number of intervals given by $\left\{y_{i}\right\}$. Let $\left\{\tilde{u}_{i}\right\}$ denote the values that $u^{n+1 / 3}$ takes; that is, $\tilde{u}_{i}$ and $\tilde{u}_{i+1}$ denote the left and right limits, respectively, of $u^{n+1 / 3}$ at the discontinuity point $x=y_{i}$. Choose positions $\left\{\bar{x}_{i}\right\}$ such that $y_{i}$ is located somewhere in the interval $\left\langle\bar{x}_{i}, \bar{x}_{i+1}\right\rangle$. Then define the residual flux function (suppressing dependency on $n$ ) by

$$
f_{\text {res }}^{\delta}(x, u)= \begin{cases}f^{\delta}(u)-f_{c}^{\delta}\left(u ; \tilde{u}_{i}, \tilde{u}_{i+1}, V^{\Delta}\right), & \text { for } x \in\left[\bar{x}_{i}, \bar{x}_{i+1}\right\rangle \text { and } u \in\left[\tilde{u}_{i}, \tilde{u}_{i+1}\right] \\ 0, & \text { for } x \in\left[\bar{x}_{i}, \bar{x}_{i+1}\right\rangle \text { and } u \notin\left[\tilde{u}_{i}, \tilde{u}_{i+1}\right] .\end{cases}
$$

Introduce a small positive parameter $\tau$, later referred to as the correction time, and let $v_{i}(x, t)$ denote the front tracking solution to the (local) residual conservation law

$$
\frac{\partial}{\partial t} v_{i}+V^{\Delta}(x, t) \frac{\partial}{\partial x} f_{\text {res }}^{\delta}\left(\bar{x}_{i}+, v_{i}\right)=0, \quad(x, t) \in\left[\bar{x}_{i}, \bar{x}_{i+1}\right\rangle \times\langle 0, \tau]
$$

with data $\left.v_{i}\right|_{t=0}=u_{-}^{n+2 / 3}$ restricted to $\left[\bar{x}_{i}, \bar{x}_{i+1}\right\rangle$. Connect the solutions of (18) for all $i$ and let $v(x, t)$ denote this solution, which is defined for all $x \in \mathbb{R}$. If $\mathcal{P}_{\Delta, \tau}(t)$ denotes the operator that maps $u^{n+1 / 3}$ to $v(x, \tau)$, we define $u^{n+2 / 3}=\mathcal{P}_{\Delta, \tau}(\Delta t) u^{n+1 / 3}$.

Step 3 (reaction/source/sink): Let $z_{\Delta}(x, t)=\mathcal{R}_{\Delta}(t) u^{n+2 / 3}$ be the solution of

$$
\frac{\partial}{\partial t} z=Q(x, z), \quad z(x, 0)=u^{n+2 / 3}(x), \quad(x, t) \in \mathbb{R} \times\langle 0, \Delta t]
$$

computed by the forward-Euler scheme using $N_{r}$ time steps of length $k_{r}$, i.e, $N_{r} k_{r}=\Delta t$. Finally, define the splitting solution at time $t=t_{n+1}$ by $u^{n+1}=\mathcal{R}_{\Delta}(\Delta t) u^{n+2 / 3}$. 
The splitting solution $\left\{u^{n}\right\}_{n=0}^{N}$ is constructed inductively. First, set $u^{0}=\pi u_{0}$ and then compute $u^{n+1}$ from $u^{n}$ for all $0 \leq n<N$ using the product formula

$$
u^{n+1}=\left[\mathcal{R}_{\Delta}(\Delta t) \circ \mathcal{P}_{\Delta, \tau}(\Delta t) \circ \mathcal{S}_{\Delta}(\Delta t)\right] u^{n} .
$$

Step 2 (diffusion revisited): Instead of having a separate correction step, the residual flux term can be included in the equation modelling diffusion. We then get the following parabolic equation

$$
\frac{\partial}{\partial t} w+V^{\Delta}(x, t) \frac{\partial}{\partial x} f_{\text {res }}^{\delta}(x, w)=\varepsilon \frac{\partial^{2}}{\partial x^{2}} w, \quad(x, t) \in \mathbb{R} \times\langle 0, \Delta t],
$$

that must be solved with data $\left.w\right|_{t=0}=u^{n+1 / 3}$. It is not difficult to modify the difference scheme (13) so that it takes into account the residual term $f_{\text {res }}^{\delta}$. To this end, consider a fixed interval $\left\langle\bar{x}_{i}, \bar{x}_{i+1}\right\rangle$ on which $f_{\text {res }}^{\delta}$ is nonzero (on intervals where $f_{\text {res }}^{\delta}$ is zero, the scheme is not altered). For notational convenience we let $g$ denote this residual term. Restricted to the $i$ th interval, we compute the solution according to the following modified difference formula

$$
w_{j}^{m+1}=-\beta_{d} V^{\Delta}\left(x_{j}, t_{m}\right)\left(g_{j+1}^{m}-g_{j-1}^{m}\right)+\alpha_{d} w_{j-1}^{m}+\left(1-2 \alpha_{d}\right) w_{j}^{m}+\alpha_{d} w_{j+1}^{m},
$$

for all $j$ such that $x_{j} \in\left\langle\bar{x}_{i}, \bar{x}_{i+1}\right\rangle$. Here $\beta_{d}=k_{d} /(2 h)$ and $g_{j}^{n}=g\left(w_{j}^{n}\right)$. Recall that (22) is stable provided $\alpha_{d} \leq 1 / 2$ and $h \leq 2 \varepsilon\left\|g^{\prime}\right\|_{\infty}$. Consult, for example, [24] for a discussion of this scheme when $V^{\Delta}=1$. In applications a more sophisticated scheme should of course be used; we have successfully applied a Petrov-Galerkin finite element method [29] instead of (22). The advantage with (21) is that we avoid choosing the parameter $\tau$, but at the expense of solving a more complicated diffusion equation. If $\mathcal{P}_{\Delta}(t)$ denotes the difference operator associated with (21), we hence introduce the alternative corrected splitting scheme

$$
u^{n+1}=\left[\mathcal{R}_{\Delta}(\Delta t) \circ \mathcal{P}_{\Delta}(\Delta t) \circ \mathcal{S}_{\Delta}(\Delta t)\right] u^{n} .
$$

\section{Convergence}

It is well known that there exists a unique classical solution $u(x, t)$ to the initial-value problem (1). For $t>0$ the solution has all the continuous derivatives occurring in the equation. Furthermore, the initial data is in general assumed in the weak sense, i.e.,

$$
\int\left(u(x, t)-u_{0}(x)\right) \phi(x) d x \rightarrow 0, \quad t \rightarrow 0+, \quad \forall \phi \in C_{0}(\mathbb{R}) .
$$

We recall that a function $u(x, t)$ is called a weak or generalized solution to (1) provided

$$
\iint_{Q_{T}}\left(u \frac{\partial}{\partial t} \phi+F(x, t, u) \frac{\partial}{\partial x} \phi+\varepsilon u \frac{\partial^{2}}{\partial x^{2}} \phi-G(x, t, u) \phi\right) d t d x+\left.\int u_{0} \phi\right|_{t=0} d x=0,
$$

for all test functions $\phi \in C_{0}^{\infty}\left(Q_{T}\right)$ with $\left.\phi\right|_{t=T} \equiv 0$. Moreover, the weak solution $u(x, t)$ is unique and possesses for $t>0$ all the continuous derivatives occurring in (1) and satisfies this equation. Consequently, the notion of classical and weak solutions coincide. All these claims are rigorously proved by Oleinik [38]. 
We will now show that a sequence of splitting solutions is compact in the $L_{1}$ topology. For simplicity we first assume that the residual term $f_{\text {res }}$ is zero, and then towards the end of this section discuss the case with a nonzero residual flux term. The splitting scheme in our analysis then takes the form

$$
u^{n+1}=\left[\mathcal{R}_{\Delta}(\Delta t) \circ \mathcal{H}_{\Delta}(\Delta t) \circ \mathcal{S}_{\Delta}(\Delta t)\right] u^{n} .
$$

By making appropriate assumptions (see Theorem 3.1) on $V(x, t), f(u)$, and $G(x, t, u)$, we obtain the following bounds on the source term $Q(x, t, u)=G(x, t, u)-f(u) V_{x}(x, t)$ :

$$
|Q(x, t, u)| \leq Q_{\infty}, \quad T V(Q(\cdot, t, u)) \leq Q_{V}, \quad\|Q(x, t, \cdot)\|_{L i p} \leq Q_{L},
$$

where $Q_{\infty}, Q_{v}$, and $Q_{L}$ are finite constants independent of $x, t$, and $u$. We shall in what follows assume (for convenience) that $h=\mathcal{O}(1) \Delta t$.

Lemma 3.1. The splitting solution $u^{n}$ is bounded; that is, $\left\|u^{n}\right\|_{\infty} \leq M_{1}(T)$ for some constant $M_{1}(T)$ independent of $h, \Delta t, \delta$, and $n$.

Proof. Fix an integer $l<n$. By construction we know that the solution operator $\mathcal{S}_{\Delta}(t)$ does not introduce new extrema and neither does $\mathcal{H}_{\Delta}(t)$. The latter is true due to the convex nature of the finite difference scheme (13) and because $\pi$ is the simple grid cell averaging operator. Furthermore, the form of $\mathcal{R}_{\Delta}(t)$ gives that $\left\|z^{m+1}\right\|_{\infty} \leq\left\|z^{m}\right\|_{\infty}+k_{r} Q_{\infty}$, for each $m \leq N_{r}$. Induction on $m$ and $l$ implies that $\left\|u^{n}\right\|_{\infty} \leq\left\|u_{0}\right\|_{\infty}+n \Delta t Q_{\infty} \leq M_{1}(T)$.

Lemma 3.2. The total variation of the splitting solution $u^{n}$ is finite; that is, $T V\left(u^{n}\right) \leq$ $M_{2}(T)$ for some constant $M_{2}(T)$ independent of $h, \Delta t, \delta$, and $n$.

Proof. Fix an integer $l<n$. Let us first consider the front tracking operator $\mathcal{S}_{\Delta}(t)$. The variation only changes when shock fronts (discontinuity lines) from neighbouring Riemann problems collide. Suppose that two or more fronts collide and let $u_{1}, \ldots, u_{K}$ be the values that meet in the collision. After the collision, there will be at least one new shock front, unless the values $u_{1}$ and $u_{K}$ are equal. When $u_{1}=u_{K}$, all the intermediate values disappear and the total variation decreases in the collision. If the collision produces new shocks, $u_{1}$ will be the value to the left of the leftmost shock and $u_{K}$ the value to the right of the rightmost shock, and the values in between will form a monotone sequence. Accordingly, all of the values $u_{2}, \ldots, u_{K-1}$ that are not between $u_{1}$ and $u_{K}$ disappear and the total variation decreases. Consequently, we can conclude that $T V\left(\mathcal{S}_{\Delta}(\Delta t) u^{l}\right) \leq T V\left(u^{l}\right)$.

The operator $\pi$ does not increase the total variation. By using the convex nature of (13), it easily follows that $T V\left(\mathcal{H}_{\Delta}(\Delta t) u^{l+1 / 3}\right) \leq T V\left(u^{l+1 / 3}\right)$. Let us finally look at the reaction operator $\mathcal{R}_{\Delta}(t)$. Fix an integer $m<N_{r}$. We then readily get

$$
\begin{gathered}
\sum_{i}\left|z_{i+1}^{m+1}-z_{i}^{m+1}\right| \leq \sum_{i}\left|z_{i+1}^{m}-z_{i}^{m}\right|+k_{r} \sum_{i}\left|Q\left(x_{i+1}, t_{m}, z_{i+1}^{m}\right)-Q\left(x_{i}, t_{m}, z_{i+1}^{m}\right)\right| \\
+k_{r} \sum_{i}\left|Q\left(x_{i}, t_{m}, z_{i+1}^{m}\right)-Q\left(x_{i}, t_{m}, z_{i}^{m}\right)\right|,
\end{gathered}
$$

which implies that $T V\left(z^{m+1}\right) \leq\left(1+k_{r} Q_{L}\right) T V\left(z^{m}\right)+k_{r} Q_{V}$. This can be further expanded as $T V\left(z^{m+1}\right) \leq\left(1+k_{r} Q_{L}\right)^{m} T V\left(z^{0}\right)+m k_{r} Q_{V}\left(1+k_{r} Q_{L}\right)^{m}$. Consequently, the splitting 
solution $u^{l+1}=\mathcal{R}_{\Delta}(\Delta t) u^{l+2 / 3}$ at time level $t=(l+1) \Delta t$ satisfies

$$
T V\left(u^{l+1}\right) \leq e^{\Delta t Q_{L}} T V\left(u^{l+2 / 3}\right)+\Delta t e^{\Delta t Q_{L}} Q_{V} \leq e^{\Delta t Q_{L}} T V\left(u^{l}\right)+\Delta t e^{\Delta t Q_{L}} Q_{V} .
$$

By induction on $l$ we get $T V\left(u^{n}\right) \leq e^{n \Delta t Q_{L}} T V\left(u_{0}\right)+n \Delta t e^{n \Delta t Q_{L}} Q_{V} \leq M_{2}(T)$.

Lemma 3.3. The splitting solution $u^{n}$ is $L_{1}$ Hölder continuous in the time variable with exponent $\frac{1}{2}$; that is, $\left\|u^{p}-u^{q}\right\|_{1} \leq M_{3}(T) \sqrt{|(p-q) \Delta t|}$ for some constant $M_{3}(T)$ independent of $h, \Delta t, \delta, q$, and $p$.

Proof. Fix an integer $l$ between $q$ and $p, q<p$. Since all waves in the front tracking solution have finite speed of propagation and the total variation is finite, it follows that $\left\|\mathcal{S}_{\Delta}(\Delta t) u^{l}-u^{l}\right\|_{1}=\mathcal{O}(1) \Delta t$. Moreover, using (26) it is easy to see that the reaction operator $\mathcal{R}_{\Delta}(t)$ obeys the bound $\left\|\mathcal{R}_{\Delta}(\Delta t) u^{l+2 / 3}-u^{l+2 / 3}\right\|_{1}=\mathcal{O}(1) \Delta t$. It is well known that the solution of the difference equation (13) is merely $L_{1}$ Hölder continuous in time with exponent $\frac{1}{2}$ (consult e.g. [24, section 2]); i.e., $\sum_{i}\left|w_{i}^{N_{d}}-w_{i}^{0}\right| h=\mathcal{O}(1) \sqrt{\Delta t}$. Taking into account the projection error $\left\|u^{l+1 / 3}-\pi u^{l+1 / 3}\right\|_{1}=\mathcal{O}(1) h$, we get the following strong $L_{1}$ Hölder continuity result for the approximate heat operator

$$
\left\|\mathcal{H}_{\Delta}(\Delta t) u^{l+1 / 3}-u^{l+1 / 3}\right\|_{1}=\mathcal{O}(1)(\sqrt{\Delta t}+h)=\mathcal{O}(1) \sqrt{\Delta t}
$$

Since the difference solution is only $L_{1}$ Hölder continuous, we first need to establish a weak Lipschitz continuity result of the finite difference solution. To this end, let $\phi(x)$ be a test function (with compact support) and $\phi_{h}(x)$ its piecewise constant approximation; $\phi_{h}(x)=\sum_{i} \phi_{i} \chi_{\left[x_{i}, x_{i+1}\right]}(x)$, where $\phi_{i}=\phi\left(x_{i}\right)$. Using the difference equation (13) and summation by parts, we get

$$
\begin{gathered}
\left|\sum_{i} \phi_{i}\left(w_{i}^{N_{d}}-w_{i}^{0}\right) h\right|=\left|\sum_{m, i} \phi_{i}\left(w_{i}^{m+1}-w_{i}^{m}\right) h\right|=\left|\sum_{m, i} h \phi_{i}\left[\alpha_{d}\left(w_{i-1}^{m}-2 w_{i}^{m}+w_{i+1}^{m}\right)\right]\right| \\
=\alpha_{d} h\left|\sum_{m, i} \phi_{i}\left(w_{i-1}^{m}-2 w_{i}^{m}+w_{i+1}^{m}\right)\right|=\alpha_{d} h\left|\sum_{m, i}\left(\phi_{i+1}-\phi_{i}\right)\left(w_{i+1}^{m}-w_{i}^{m}\right)\right| \\
\leq \alpha_{d} h^{2} N_{d}\left\|\phi_{x}\right\|_{\infty} \sup _{m} \sum_{i}\left|w_{i+1}^{m}-w_{i}^{m}\right|=\mathcal{O}(1) \Delta t\left\|\phi_{x}\right\|_{\infty},
\end{gathered}
$$

where we also have used Lemma 3.2. From this inequality, we get the weak estimate

$$
\begin{aligned}
\mid \int \phi & \left(\mathcal{H}_{\Delta}(\Delta t) u^{l+1 / 3}-u^{l+1 / 3}\right) d x \mid \\
& \leq\left|\int \phi_{h}\left(\mathcal{H}_{\Delta}(\Delta t) u^{l+1 / 3}-u^{l+1 / 3}\right) d x\right|+\left|\int\left(\phi-\phi_{h}\right)\left(\mathcal{H}_{\Delta}(\Delta t) u^{l+1 / 3}-u^{l+1 / 3}\right) d x\right| \\
& \leq\left|\sum_{i} \phi_{i}\left(w_{i}^{N_{d}}-w_{i}^{0}\right) h\right|+\mathcal{O}(1) h\|\phi\|_{\infty}+h\left\|\phi_{x}\right\|_{\infty}\left\|\mathcal{H}_{\Delta}(\Delta t) u^{l+1 / 3}-u^{l+1 / 3}\right\|_{1} \\
& =\mathcal{O}(1) \Delta t\left(\|\phi\|_{\infty}+\left\|\phi_{x}\right\|_{\infty}\right)+\mathcal{O}(1)\left\|\phi_{x}\right\|_{\infty} h \sqrt{\Delta t}=\mathcal{O}(1) \Delta t\left(\|\phi\|_{\infty}+\left\|\phi_{x}\right\|_{\infty}\right) .
\end{aligned}
$$


We thus conclude that

$$
\left|\int \phi\left(u^{p}-u^{q}\right) d x\right|=\mathcal{O}(1)(p-q) \Delta t\left(\|\phi\|_{\infty}+\left\|\phi_{x}\right\|_{\infty}\right)
$$

This weak estimate can now be interpolated into a strong $L_{1}$ Hölder estimate with exponent $\frac{1}{2}$ along the lines of [31]. This concludes our proof of the lemma.

Now we need to consider functions defined in the interval $\langle 0, T]$, and not merely on the time-strips $t=t_{n}$. To accomplish this, define the sequence $\left\{u_{\eta}(x, t)\right\}_{\eta>0}$ by

$$
u_{\eta}(x, t)= \begin{cases}\mathcal{S}_{\Delta}\left(2\left(t-t_{n}\right)\right) u^{n}, & t \in\left\langle t_{n}, t_{n+1 / 2}\right\rangle, \\ \mathcal{H}_{\Delta}\left(2\left(t-t_{n+1 / 2}\right)\right) u^{n+1 / 3}, & t \in\left[t_{n+1 / 2}, t_{n+1}\right\rangle, \\ \mathcal{R}_{\Delta}(\Delta t) u^{n+2 / 3}, & t=t_{n+1},\end{cases}
$$

where $u^{n+1 / 3}$ and $u^{n+2 / 3}$ are as defined above and $\eta=(h, \Delta t, \delta)$. Note that $u_{\eta}\left(x, t_{n}\right)=u^{n}$ for all $n$. This method of extending $u^{n}$ to a function defined for all $t>0$ is motivated by [8] and [31]. In view of the previous lemmas, we conclude that there exists a finite constant $M=M(T)$ (independent of $\eta$ ) such that $u_{\eta}(x, t)$ obeys:

$$
\left\|u_{\eta}(\cdot, t)\right\|_{\infty} \leq M, \quad T V\left(u_{\eta}(\cdot, t)\right) \leq M, \quad\left\|u_{\eta}\left(\cdot, t_{2}\right)-u_{\eta}\left(x, t_{1}\right)\right\|_{1} \leq M \sqrt{\left|t_{2}-t_{1}\right|} .
$$

Now a standard application of Helly's theorem (see e.g. [38]) yields the desired $L_{1}^{\text {loc }}\left(Q_{T}\right)$ convergence of a subsequence, still denoted by $\left\{u_{\eta}\right\}$, to a function $u$. We finally justify the term "approximate solution" by proving that $u(x, t)$ is the (classical) solution of (1).

Lemma 3.4. The limit $u(x, t)$ is the classical solution of the Cauchy problem (1).

Proof. Let $\mathcal{L}_{\phi}(u)$ denote the left-hand side of $(24)$. It then suffices to prove that $\mathcal{L}_{\phi}(u)=0$ for all proper test functions $\phi$ with $\left.\phi\right|_{t=T}=0$. To this end, let $v_{n}(t)=\mathcal{S}_{\Delta}(t) u^{n}, t \in\langle 0, \Delta t]$, and define a new test function $\varphi$ by $\varphi(x, t)=\phi(x, t / 2)$. Then, since $v_{n}(t)$ is an exact solution to the (perturbed) conservation law (15), the following equality holds

$$
\begin{aligned}
\iint_{t_{n}}^{t_{n+1 / 2}} & \left(\frac{1}{2} u_{\eta} \frac{\partial}{\partial t} \phi+f^{\delta}\left(u_{\eta}\right) \frac{\partial}{\partial x}\left(V^{\Delta}(x, t) \phi\right)\right) d t d x \\
= & \frac{1}{2} \iint_{0}^{\Delta t}\left(v_{n}(\xi) \frac{\partial}{\partial \tau} \varphi+f^{\delta}\left(v_{n}(\xi)\right) \frac{\partial}{\partial x}\left(V^{\Delta}(x, t) \phi\right)\right) d \xi d x \\
= & \left.\frac{1}{2} \int u^{n+1 / 3} \phi\right|_{t=t_{n+1 / 2}} d x-\left.\frac{1}{2} \int u^{n} \phi\right|_{t=t_{n}} d x
\end{aligned}
$$

where we have used the substitution $\xi=2\left(t-t_{n}\right)$. Let $\left\{t_{m}\right\}$ denote the local time levels associated with the time step $k_{d}$ and let $\phi_{i}^{m}=\phi\left(x_{i}, t_{m}\right)$. Multiplying the finite difference equation (13) by $\phi_{i}^{m} k_{d} h$ and applying summation by parts, we get

$$
\begin{array}{r}
\sum_{i} \sum_{m<N_{d}}\left(w_{i}^{m}\left[\frac{\phi_{i}^{m+1}-\phi_{i}^{m}}{k_{d}}\right]+\varepsilon w_{i}^{m}\left[\frac{\phi_{i-1}^{m}-2 \phi_{i}^{m}+\phi_{i+1}^{m}}{h^{2}}\right]\right) k_{d} h \\
=\sum_{i} w_{i}^{N_{d}} \phi_{i}^{N_{d}} h-\sum_{i} w_{i}^{0} \phi_{i}^{0} h, \quad w_{i}^{0}=\left(\pi u^{n+1 / 3}\right)\left(x_{i}\right) .
\end{array}
$$


Letting $w_{\Delta}(x, t)=\mathcal{H}_{\Delta}(\Delta t) u^{n+1 / 3}$, we can obviously replace this equality by

$$
\begin{aligned}
\iint_{0}^{\Delta t} & \left(w_{\Delta} \frac{\partial}{\partial t} \phi+\varepsilon w_{\Delta} \frac{\partial^{2}}{\partial x^{2}} \phi\right) d t d x= \\
& \left.\int u^{n+2 / 3} \phi\right|_{t=\Delta t} d x-\left.\int u^{n+1 / 3} \phi\right|_{t=0} d x+\left.\int\left(u^{n+1 / 3}-\pi u^{n+1 / 3}\right) \phi\right|_{t=0} d x
\end{aligned}
$$

making an allowable error of $\mathcal{O}(1)\left(\Delta t h+\Delta t k_{d}\right)=\mathcal{O}(1) \Delta t^{2}$. As in (28), we deduce

$$
\begin{aligned}
& \iint_{t_{n+1 / 2}}^{t_{n+1}}\left(\frac{1}{2} u_{\eta} \frac{\partial}{\partial t} \phi+\varepsilon u_{\eta} \frac{\partial^{2}}{\partial x^{2}} \phi\right) d t d x= \\
& \left.\quad \frac{1}{2} \int\left(u_{\eta} \phi\right)\right|_{t=t_{n+1}^{-}} d x-\left.\frac{1}{2} \int u^{n+2 / 3} \phi\right|_{t=t_{n+1 / 2}} d x+\left.\int\left(u^{n+1 / 3}-\pi u^{n+1 / 3}\right) \phi\right|_{t=t_{n+1 / 2}} d x .
\end{aligned}
$$

Having in mind (14) and (26), a straightforward calculation yields

$$
\left.\int\left(u_{\eta} \phi\right)\right|_{t=t_{n+1}^{-}} d x=\left.\int u^{n+1} \phi\right|_{t=t_{n+1}} d x-\iint_{t_{n+1 / 2}}^{t_{n+1}} Q\left(x, t, u_{\eta}\right) \phi d t d x
$$

within an allowable error of $\mathcal{O}(1) \Delta t^{3 / 2}$. If we now add the approximate weak formulations for $u_{\eta}(x, t)$ on $\left\langle t_{n}, t_{n+1 / 2}\right\rangle$ and $\left\langle t_{n+1 / 2}, t_{n+1}\right]$, and sum over $n$, the resulting global weak formulation, now within an allowable error of $\mathcal{O}(1) \sqrt{\Delta t}$, takes the form

$$
\begin{aligned}
& \iint_{Q_{T}}\left(\frac{1}{2} u_{\eta} \frac{\partial}{\partial t} \phi+S_{N}(t) f^{\delta}\left(u_{\eta}\right) \frac{\partial}{\partial x}\left(V^{\Delta}(x, t) \phi\right)+T_{N}(t) \varepsilon u_{\eta} \frac{\partial^{2}}{\partial x^{2}} \phi+T_{N}(t) Q\left(x, t, u_{\eta}\right) \phi\right) d t d x \\
& 29) \quad+\left.\frac{1}{2} \int u_{0} \phi\right|_{t=0} d x=\left.\sum_{n=0}^{N-1} \int\left(u^{n+1 / 3}-\pi u^{n+1 / 3}\right) \phi\right|_{t=t_{n+1 / 3}} d x .
\end{aligned}
$$

Here $S_{N}(t)$ and $T_{N}(t)$ denote the characteristic functions

$$
S_{N}(t)=\sum_{n=0}^{N-1} \chi_{\langle n \Delta t,(n+1 / 2) \Delta t\rangle}(t), \quad T_{N}(t)=\sum_{n=0}^{N-1} \chi_{[(n+1 / 2) \Delta t,(n+1) \Delta t]}(t) .
$$

Note that $S_{N}(t)$ and $T_{N}(t)$ both tend weakly in $L_{2}$ to $1 / 2$. We assume that $f^{\delta}$ and $V^{\Delta}$ are chosen such that $\left\|f-f^{\delta}\right\|_{W^{1, \infty}} \rightarrow 0$ and $\left\|V-V^{\Delta}\right\|_{W^{1, \infty}} \rightarrow 0$ as $\eta \rightarrow 0$. Furthermore, the global projection error (the right-hand side of the equality (29)) tends to zero as $\eta \rightarrow 0$ (see e.g. [31]). In view of the dominated convergence theorem, we can thus pass to the limit as $\eta \rightarrow 0$ in (29), obtaining (remember that $Q=G-f V_{x}$ )

$$
\mathcal{L}_{\phi}(u)=\lim _{\eta \rightarrow 0} \mathcal{L}_{\phi}\left(u_{\eta}\right)=0
$$

where $u=\lim _{\eta \rightarrow 0} u_{\eta}$. This concludes the proof of the lemma.

Consequently, we have the following main theorem. 
Theorem 3.1. Let $F(x, t, u)=V(x, t) f(u)$ be the flux term and $G(x, t, u)$ the reaction term. Suppose that $V, f$, and $G$ are bounded and smooth functions of all their arguments. In addition, suppose that $G(\cdot, t, u)$ and $V_{x}(\cdot, t)$ have bounded variation and that the initial data $u_{0}$ is a function of bounded variation. Then the operator splitting solutions (25) converge to the unique classical solution of the Cauchy problem

$$
\frac{\partial}{\partial t} u+\frac{\partial}{\partial x} F(x, t, u)=\varepsilon \frac{\partial^{2}}{\partial x^{2}} u+G(x, t, u), \quad u(x, 0)=u_{0}(x), \quad(x, t) \in Q_{T} .
$$

Let us now consider the corrected splitting formulas (20) and (23), starting with (20). Assume for simplicity that there is only one residual flux term and name it $g$. Let $v(x, t)$ denote the solution of the corresponding residual conservation law (18), which is to be understood, of course, in the sense of measures and defined on $\mathbb{R} \times\langle 0, \tau]$, assuming that $\tau=\mathcal{O}(1) \Delta t$. Recall that $v(x, t)$ depends on the discretization parameters $\eta=(h, \Delta t, \delta)$. Lemmas 3.1, 3.2, and 3.3 are still valid and thus the corrected splitting solutions (20) are compact in $L_{1}^{\text {loc }}\left(Q_{T}\right)$. In view of Lemma 3.4, it is sufficient to show that the measure $g(v)_{x}$ is "small" (in a proper sense) to conclude that the limit $u(x, t)$ is the desired solution. The next lemma says that this term can in fact be viewed as an allowable error term.

Lemma 3.5. Let $\phi \in C_{0}^{\infty}(\mathbb{R})$ and let $\Phi(x, t)=\phi(x) V^{\Delta}(x, t)$ (consult (18)). Then we have

$$
\left|\int \Phi(x, t) d \mu_{\eta}\right|=\mathcal{O}(1) \sqrt{\Delta t}, \quad d \mu_{\eta}=\frac{\partial}{\partial x} g(v) d x
$$

Proof. Observe first that the measure $g(v)_{x}$ has finite variation because of Lemma 3.2, and that $\Phi_{x}$ is uniformly bounded independently of $\eta$. Now a classical $B V$ argument (consult e.g., [43]) allows us to calculate as follows

$$
\begin{aligned}
\left|\int \Phi(x) d \mu_{\eta}\right| & \leq\left\|\frac{\partial}{\partial x} \Phi\right\| \int|g(v)| d x=\mathcal{O}(1) \int\left|g(v)-g\left(u^{n+1 / 3}\right)\right| d x \\
& =\mathcal{O}(1) \int\left|v(x, t)-u^{n+1 / 3}(x)\right| d x=\mathcal{O}(1) \sqrt{\Delta t}
\end{aligned}
$$

where we have used the key property $g\left(u^{n+1 / 3}\right) \equiv 0$ for all $n$, the Lipschitz continuity of $g$, and Lemma 3.3. This concludes the proof.

It is not difficult, by mimicking the proofs of Lemmas 3.1, 3.2, and 3.3, to also get compactness of the corrected splitting solutions given by (23). Again, to conclude that the limit $u(x, t)$ is the solution of our problem, it suffices to show that the term in the difference scheme (21) associated with the residual flux $g$ is within an allowable error.

Lemma 3.6. Let $\phi \in C_{0}^{\infty}(\mathbb{R})$ and let $\Phi_{j}^{m}=\phi\left(x_{j}\right) V^{\Delta}\left(x_{j}, t_{m}\right)$ (consult $(22)$ ). Then we have

$$
\left|\sum_{j} \Phi_{j}^{m}\left[\frac{g_{j+1}^{m}-g_{j-1}^{m}}{2 h}\right] h\right|=\mathcal{O}(1) \sqrt{\Delta t}
$$


Proof. The proof is a discrete version of the proof of Lemma 3.5. Applying summation by parts and Lemma 3.3, we get

$$
\begin{aligned}
\left|\sum_{j} \Phi_{j}^{m}\left[\frac{g_{j+1}^{m}-g_{j-1}^{m}}{2 h}\right] h\right| & =\left|\sum_{j}\left[\frac{\Phi_{j+1}^{m}-\Phi_{j-1}^{m}}{2 h}\right] g_{j}^{m} h\right| \leq \| \frac{\partial}{\partial x} \Phi|| \sum_{j}\left|g_{j}^{m}\right| h \\
& =\mathcal{O}(1) \int\left|g\left(w_{\Delta}\left(x, t_{m}\right)\right)-g\left(u^{n+1 / 3}\right)\right| d x \\
& =\mathcal{O}(1) \int\left|w_{\Delta}\left(x, t_{m}\right)-u^{n+1 / 3}(x)\right| d x=\mathcal{O}(1) \sqrt{\Delta t}
\end{aligned}
$$

where $w_{\Delta}(x, t)$ is the piecewise constant interpolation of the difference solution (22).

Consequently, we have our second and final main theorem.

Theorem 3.2. Under the same assumptions, the conclusion of Theorem 3.1 is also valid for the corrected operator splitting formulas (20) and (23).

Of course, the above convergence theorems are also true for equations that are in nonconservative form to begin with, i.e., equations of the form

$$
\frac{\partial}{\partial t} u+V(x, t) \frac{\partial}{\partial x} f(u)=\varepsilon \frac{\partial^{2}}{\partial x^{2}} u+G(x, t, u) .
$$

\section{Numerical Examples}

We present and discuss two numerical examples which each will focus on an aspect of the method. The first example is a purely academic case, but is chosen to clearly demonstrate the effect of correction. The next is drawn from modelling of glacier movement and growth.

First, a few words on the implementation of the correction step. Although the residual term is defined for every discontinuity in the advection solution, it is zero for all steps of size $\delta$ (i.e., rarefaction waves). Similarly, it has little effect for "small" discontinuities. To increase code efficiency, one should therefore introduce a threshold which a shock in the advection solution must exceed before the corresponding residual flux term is computed.

4.1. Academic Case. In our first example we consider (2) with $f(u)=4 u^{2}\left(1-u^{2}\right)$, $V(x)=1+\nu \sin (\pi x)$, and $G \equiv 0$. This example serves the purpose of showing the schemes ability to resolve the balance of advection and diffusion correctly. Source terms will be considered in the next example. Note that the flux function is nonconvex, which represents no additional challenge due to the ability of the front tracker to solve Riemann problems for any flux function.

We will consider two different initial data, first a Riemann problem and then a highly non-monotone initial function. The Riemann problem is given by $u_{0}(x)=\operatorname{sgn}(x)$. We put $Q \equiv 0$ and use $\nu=0.75, \varepsilon=0.05$. The computational domain is $[-2,2]$.

In the computations we used a grid with 100 uniform grid cells. Figure 1 shows the solution computed by OS and the two formulations (20) and (23) of COS using one time step (i.e., a CFL number of 100). Note that the solutions are plotted as step functions, and thus do not appear as smooth as if we had used a linear interpolation. We see that both 

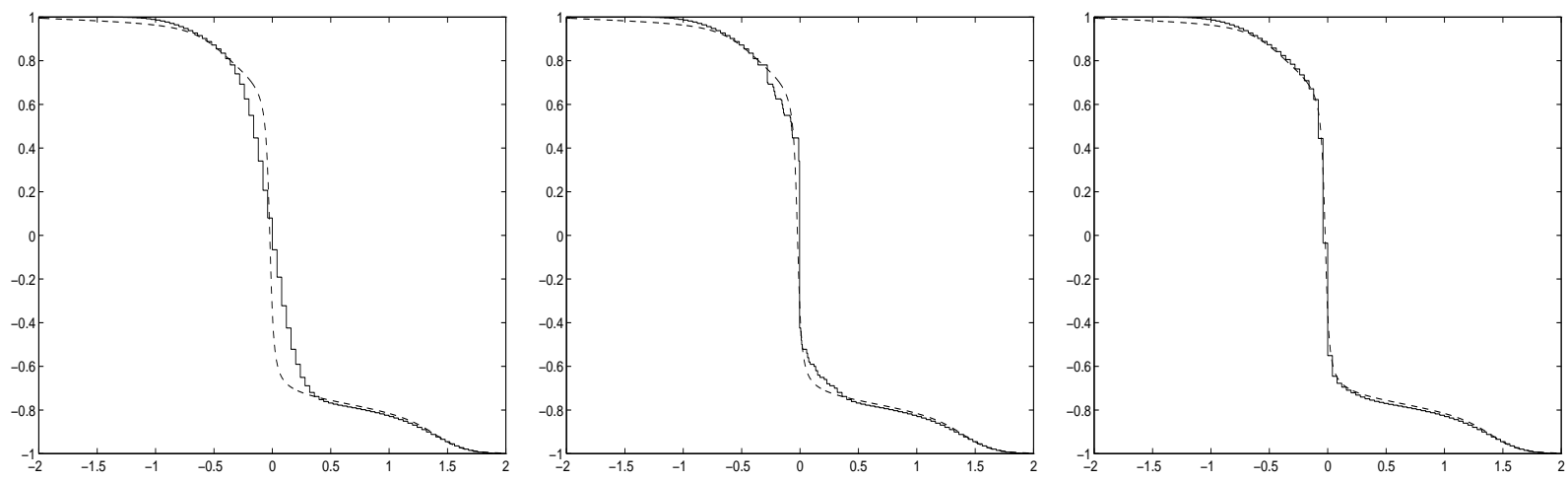

Figure 1. The reference solution (dashed) at time $t=0.3$ compared to the OS solution with one time step (left), COS with a separate correction step and $\tau=0.2 \Delta t$ (middle), and COS with a modified diffusion equation (right).
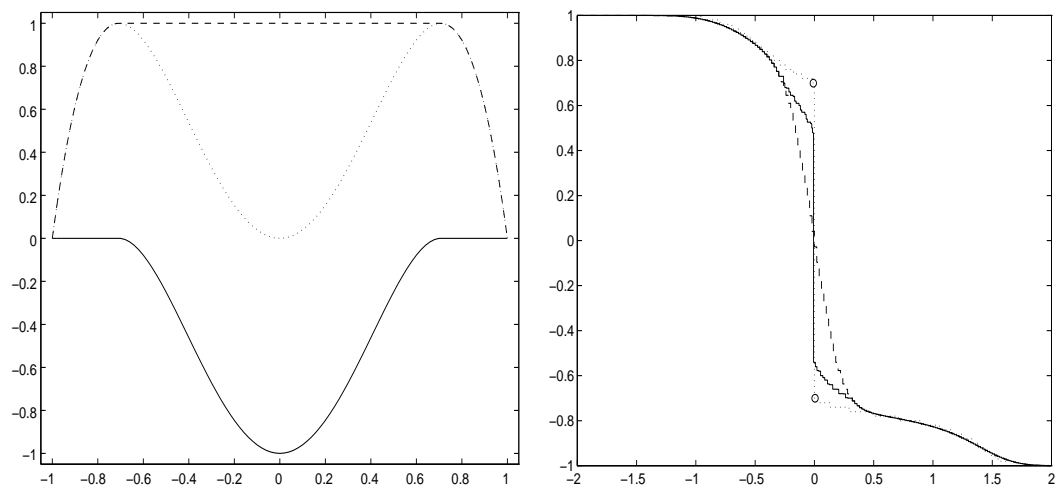

Figure 2. (Left) The flux function (dotted), upper concave envelope (dashed), and residual flux function (solid) used by COS. (Right) Intermediate solutions for COS with separate correction: advection solution (dotted), diffusion solution (dashed), and after correction (solid).

formulations of COS give notable improvement over OS, with a slight preference to COS with a modified diffusion equation. We observed that OS needed approximately 10 time steps to gain the same resolution of the shock front. In Figure 2 (left) we have given the residual flux used by COS. Figure 2 (right) shows intermediate solutions for COS. Large shocks where the correction operator is applied is indicated by circles (at $y_{i}$ values).

In the second test we use initial data $u_{0}(x)=\sin (\pi x), \nu=0.1, \varepsilon=0.02$, and $G=$ $-V^{\prime}(x) f(u)$. The computational domain is now [-5,5]. Figure 3 (left) shows a section of the solution at time $t=0.3$ computed on a grid with 200 uniform cells using three time steps (i.e., a CFL number of 17). Here COS uses $\tau=0.2 \Delta t$ and gives an improved resolution of shock fronts compared to OS. The corresponding results of COS with a modified diffusion equation (23) are similar. In Figure 3 (right) we have plotted the intermediate solutions 

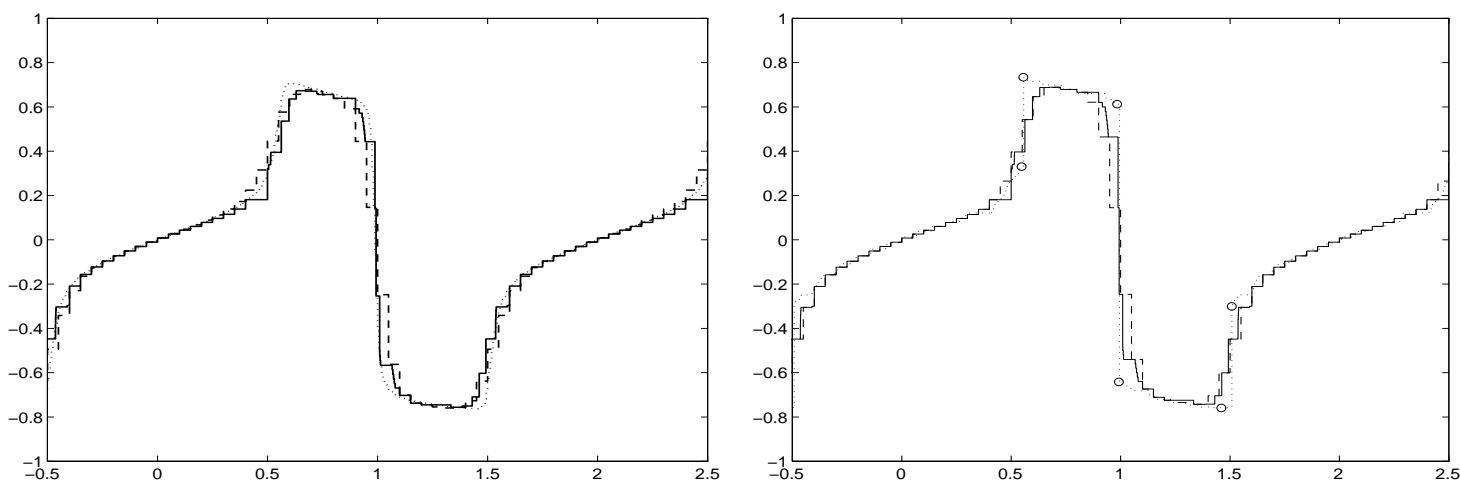

Figure 3. (Left) A section of the solution at time $t=0.3$ computed by OS (dashed) and COS (solid) together with a fine grid reference solution (dotted). (Right) Intermediate solutions for COS during the last time step.

for COS during the last time step, similarly as in Figure 2. Observe that only a few shocks in the advection solution have sufficient strength to introduce a nonzero residual flux.

The conclusion of the first example is that the correction step implies that the accuracy in shock regions is largely independent of the size of the time step, which contrasts strongly with standard splitting. The effect is even more pronounced for a larger time step $(\Delta t \gg \varepsilon)$.

4.2. Glacier Growth. In our second example we look at a simple one-dimensional model for glacier growth. Although the equations are realistic for describing a glacier, the computations are carried out for two simple examples. However, we wish to point out that our methodology is applicable for actual computations and it is currently being used in a study of Briksdalsbreen, which is a part of Jostedalsbreen (Norway), the largest glacier in continental Europe.

A glacier with height $h(x, t)$ rests upon a flat mountain, making an angle $\alpha$ with the horizontal direction, see Figure 4. Following Fowler [21], the flow is described by the conservation of mass and momentum in an incompressible flow. The stress tensor is proportional to the deformation velocity; that is, $\tau_{i j}=\mathcal{O}(1) \dot{\epsilon}_{i j}$. The deformation velocity is given by

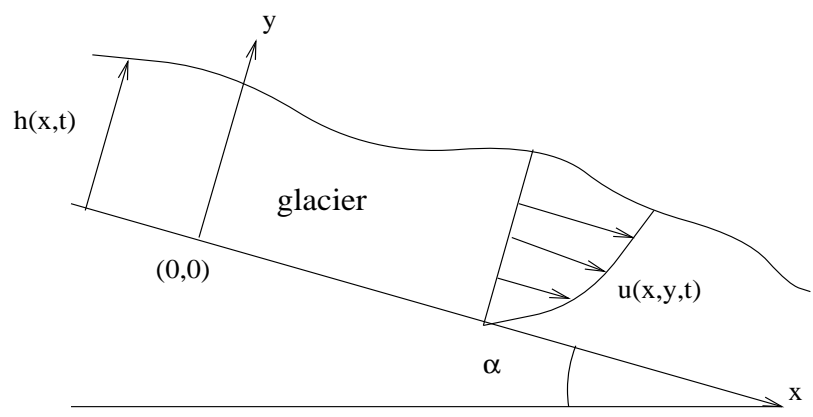

FiguRE 4. A simplified model of a glacier 

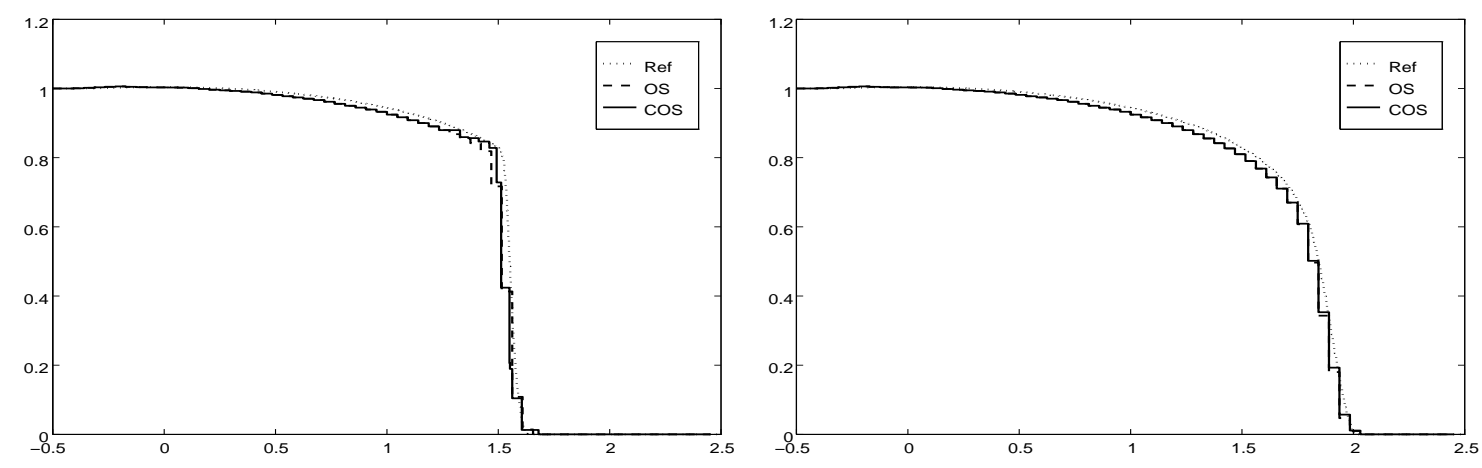

Figure 5. Snapshots of a moving glacier at times $t=2.0, t=4.0$ computed with $\mathrm{CFL}=10$. Note that the difference between OS and COS is not so pronounced as in the previous example. The reason is that $\Delta t$ is not significantly larger than $\varepsilon$.

$\dot{\epsilon}_{i j}=\frac{1}{2}\left(\frac{\partial u_{i}}{\partial x_{j}}+\frac{\partial u_{j}}{\partial x_{i}}\right)$. The glacier is a viscoplastic fluid, and the deformation is described approximately by an experimental relation called Glen's law, $\epsilon_{i j}=\frac{1}{2} A \tau^{n-1} \tau_{i j}$. The factor $A$ generally depends upon the temperature, but here we assume that it is a constant. The exponent $n$ is measured to be typically in the range $3-4$.

We now make the approximation of a shallow ice; that is, the typical length scale $l$ of the glacier is much larger than the typical depth $d$. Scaling variables and neglecting terms of lower order, Fowler derives the relation

$$
\frac{\partial u}{\partial y}=A\left[1-\varepsilon h_{x}\right]^{n}(h-y)^{n}
$$

where $\varepsilon=\frac{d}{l} \cot \alpha$ is a small parameter. Letting $u_{b}$ denote the sliding velocity at the bottom of the glacier, the volume flux becomes

$$
u_{b} h+A\left[1-\varepsilon h_{x}\right]^{n} \frac{h^{n+2}}{n+2} .
$$

The accumulation and ablation (melting) can be modelled by a source term $a(x, t, h)$. If we make the approximation that $\left(1-\varepsilon h_{x}\right)^{n} \approx 1-n \varepsilon h_{x}$, we arrive at the following equation

$$
\frac{\partial h}{\partial t}+\frac{\partial}{\partial x}\left(u_{b} h+A \frac{h^{n+2}}{n+2}\right)-\frac{\partial}{\partial x}\left(n \varepsilon A \frac{h^{n+2}}{n+2} \frac{\partial h}{\partial x}\right)=a(x, t, h) .
$$

Here the diffusion term is nonlinear and of the form $\varepsilon\left(\nu(h) h_{x}\right)_{x}$, where the function $\nu(h)$ has a one point degeneracy; that is, $\nu(0)=0$. Since nonlinear diffusion terms are not treated in this paper, we replace $\varepsilon\left(\nu(h) h_{x}\right)_{x}$ by $\varepsilon h_{x x}$ and refer instead to [18] for a treatment of the nonlinear, possibly degenerate case. This replacement does not effect the solution to a large extent, except at the foot of the front, where we now gain more regularity.

First we look at a Riemann problem with $h(x, 0)=\chi_{(-\infty, 0]}$. (A very simple model of an outlet into a valley). We are not interested in the glacier upstream and therefore use only a short interval of positive accumulation above zero $(x<0)$ and increasing ablation further downstream, We assume that the rate of accumulation and ablation (depending on 
the sign of the source term) is only dependent upon the altitude, i.e., on $x$. To model this, we use the source $a(x, t, h)=a_{0}(x)$ if $h(x, t)>0$ and $\max \left(a_{0}(x), 0\right)$ if $h(x, t)=0$, thus disregarding any seasonal variation in the accumulation,

$$
a_{0}(x)= \begin{cases}\frac{1}{2}(x+0.4), & \text { for } x \in[-0.4,-0.2], \\ -\frac{1}{2} x, & \text { for } x>-0.2, \\ 0, & \text { otherwise. }\end{cases}
$$

As a simple scaling we have used $u_{b}=\frac{1}{4}, n=4, A=\left(1-u_{b}\right)(n+2)$, and $\varepsilon=0.01$. Figure 5 shows two snapshots of the movement of the glacier front (snout). At time $t=2.0$ (left) the snout is advancing, while at $t=4.0$ (right) it has reached a stationary state where the ablation balances the forward movement. In both plots the grid is uniform with 64 grid blocks and we used 64 time steps to advance the solution up to time $t=4.0$ (corresponding to a CFL number of approximately 10). The reference solutions computed on a 1024 grid are shown as dotted lines. In both plots a small splitting error (due to the source term) is evident, giving a too low height of the glacier. At time $t=2.0$ the corrected operator splitting (solid line) resolves the shock front slightly sharper than the operator splitting (dashed line) due to the presence of strong shocks in the advection step. However, at time $t=4.0$ the glacier front is now longer sharp, and the two solutions coincide. We stress that the difference between OS and COS is not supposed to be notable in this example, since $\Delta t$ is not significantly larger than the diffusion scale $\varepsilon$.

Second, we look at the growth of a new glacier. The glacier is to be restricted approximately to the interval $[-5,5]$, and hence we use the source term,

$$
a(x, t)= \begin{cases}-0.01 x+0.05 \sin (2 \pi t), & x>-5.0, \\ 0, & x<-5.0 .\end{cases}
$$

Here the second term models seasonal variations; zero accumulation at the top in the middle of summer and no ablation at the bottom in the middle of winter. Figure 6 shows snapshots of a very accurate simulation of the glacier growth. The solution is computed by OS on a grid with 960 nodes using 8.000 time steps to reach time $t=100.0$. This corresponds to a CFL number 5 and $\Delta t \approx \varepsilon$. In Figure 7 we compare this accurate solution with COS using 80 time steps on a grid with 240 cells; that is a CFL number 132 ! Even for this extreme case COS seems able to resolve the solution fairly well. Note however, that the solution is best resolved in the beginning when the process is dominated by the accumulation. Running OS with the same parameters gave a more diffused snout during the advancing phase, but otherwise comparable results.

\section{ACKNOWLEDGEMENT}

We thank Helge K. Dahle, Magne S. Espedal, Steinar Evje, Helge Holden, and Nils Henrik Risebro for interesting discussions. We thank Harald E. Krogstad, Runar Holdahl, and Øystein Stemme for help with the glacier example. This work was carried out while the first author was a guest at the Institute for Mathematics and its Applications (IMA) 


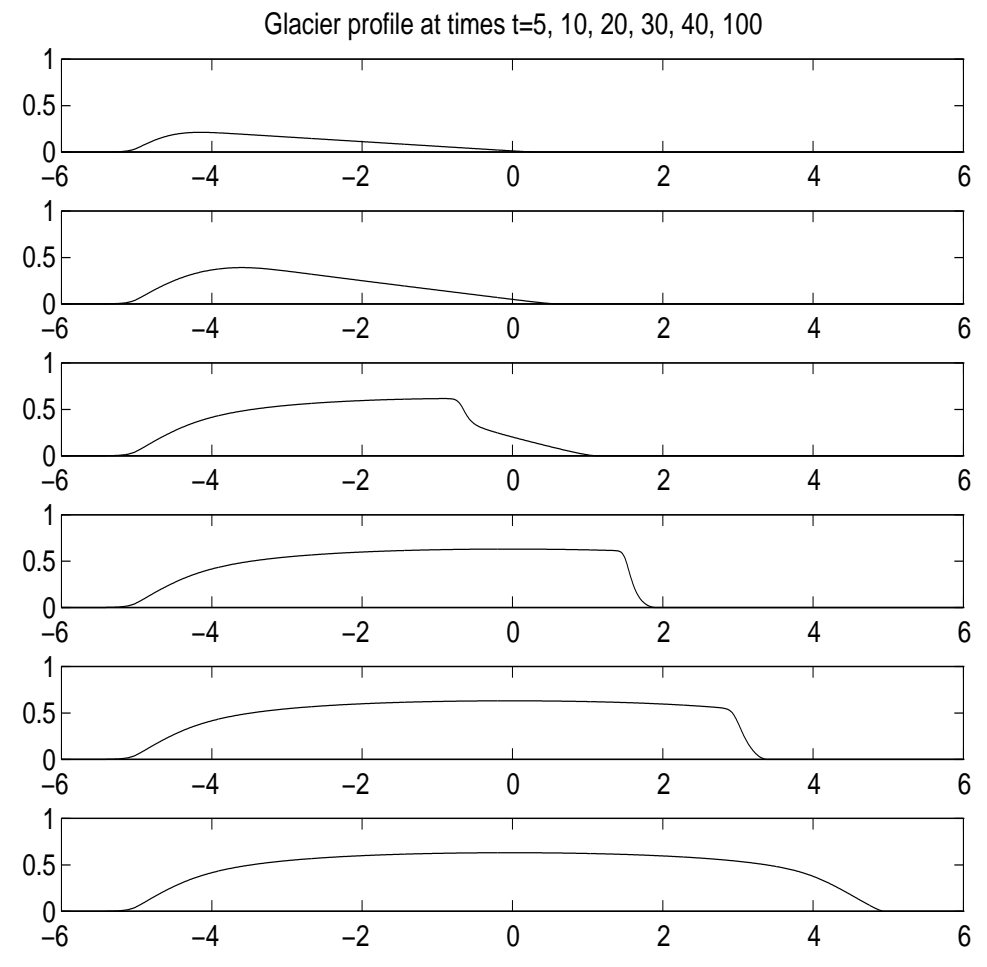

FiguRE 6. Profile of a growing glacier.
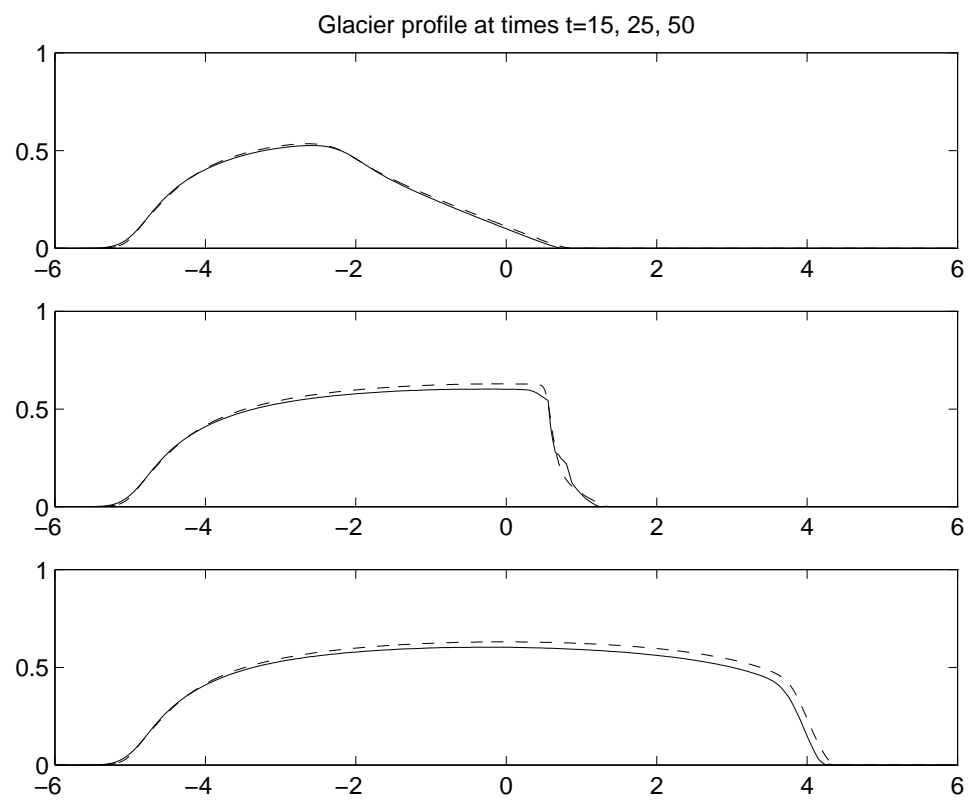

Figure 7. Profiles computed by COS with CFL number 132 on a 240 grid (solid line) compared to the very accurate solution (dashed line). 
at the University of Minnesota. This author would like to thank IMA for financial support and great hospitality.

\section{REFERENCES}

[1] T. Arbogast and M. F. Wheeler. A characteristic-mixed finite element method for advection-dominated transport problems. SIAM J. Num. Anal., 32:404-424, 1995.

[2] J. Bear and A. Verruijt. Modeling groundwater flow and pollution. Reidel, Dordrecht, 1987.

[3] F. Black and M. Scholes. The pricing of options and corporate liabilities. Jour. Pol. Econ., 81:637-659, 1973.

[4] J. M. Burgers. The nonlinear diffusion equation. Reidel, Dordrecht, 1974.

[5] M. A. Celia, T. F. Russell, I. Herrera, and R. E. Ewing. An Eulerian-Lagrangian localized adjoint method for the advection-diffusion equation. Adv. Water Resources, 13:187-205, 1990.

[6] R. Chella and J. M. Ottino. Modelling of rapidly-mixed-fast-crosslinking exothermic polymerizations. AIChE J., 29:373-382, 1983.

[7] B. Cockburn and C.-W. Shu. The local discontinuous galerkin method for time-dependent convectiondiffusion systems. Preprint, IMA, University of Minnesota, Minneapolis, 1997.

[8] M. G. Crandall and A. Majda. The method of fractional steps for conservation laws. Numer. Math., $34: 285-314,1980$.

[9] G. Crasta and B. Piccoli. Viscosity solutions and uniqueness for systems of inhomogenous balance laws. Preprint, SISSA, Italy, June 1995.

[10] C. M. Dafermos. Polygonal approximation of solutions of the initial value problem for a conservation law. J. Math. Anal. Appl., 38:33-41, 1972.

[11] H. K. Dahle. Adaptive characteristic operator splitting techniques for convection-dominated diffusion problems in one and two space dimensions. $\mathrm{PhD}$ thesis, Department of Mathematics, University of Bergen, 1988.

[12] C. N. Dawson. Godunov-mixed methods for advective flow problems in one space dimension. SIAM J. Num. Anal., 28(5):1282-1309, Oct. 1991.

[13] C. N. Dawson. High resolution upwind-mixed finite element methods for advection-diffusion equations with variable time-stepping. Numer. Meth. for PDEs, 11:525-538, 1995.

[14] C. N. Dawson, C. J. van Duijn, and M. F. Wheeler. Characteristic-Galerkin methods for contaminant transport with non-equilibrium adsorption kinetics. SIAM J. Num. Anal., 31:982-999, 1994.

[15] L. Demkowitz and J. T. Oden. An adaptive characteristic Petrov-Galerkin finite element method for convection-dominated linear and nonlinear parabolic problems in two space variables. Computer Methods in Applied Mechanics and Engineering, 55:63-87, 1986.

[16] J. Douglas and T. F. Russell. Numerical methods for convection-dominated diffusion problems based on combining the method of characteristics with finite element or finite difference procedures. SIAM J. Num. Anal., 19(5):871-885, Oct. 1982.

[17] M. S. Espedal and R. E. Ewing. Characteristic Petrov-Galerkin subdomain methods for two-phase immiscible flow. Computer Methods in Applied Mechanics and Engineering, 64:113-135, 1987.

[18] S. Evje and K. H. Karlsen. A note on viscous splitting of degenerate convection-diffusion equations. Preprint, IMA, University of Minnesota, Minneapolis, 1997. Available at the URL http://www . math. nt nu. no/conservation/.

[19] R. E. Ewing. Operator splitting and Eulerian-Lagrangian localized adjoint methods for multiphase flow. In Whiteman, editor, The Mathematics of Finite Elements and Applications VII MAFELAP, pages 215-232. Academic press, San Diego, CA, 1991.

[20] R. E. Ewing and T. F. Russell. Efficient time stepping methods for miscible displacement problems in porous media. SIAM J. Num. Anal., 19:1-67, 1982.

[21] A. C. Fowler. Glaciers and ice sheets. In J. I. Diaz, editor, The Mathematics of Models for Climatology and Environment, volume 48 of NATO ASI Series, pages 302-336. Springer Verlag, 1997. 
[22] E. Godlewski and P.-A. Raviart. Numerical approximation of hyperbolic systems of conservation laws. Springer Verlag, 1996.

[23] I. Herrera, R. E. Ewing, M. A. Celia, and T. F. Russell. Eulerian-lagrangian localized adjoint methods: The theoretical framework. Numer. Meth. for PDEs, 9:431-457, 1993.

[24] D. Hoff and J. Smoller. Error bounds for finite-difference approximations for a class of nonlinear parabolic systems. Math. Comp., 45(171):35-49, July 1985.

[25] H. Holden, L. Holden, and R. Høegh-Krohn. A numerical method for first order nonlinear scalar conservation laws in one-dimension. Comput. Math. Applic., 15(6-8):595-602, 1988.

[26] H. Holden and N. H. Risebro. Conservation laws with a random source. To appear in Applied Mathematics and Optimization. Available at the URL http://www . math.ntnu.no/conservation/.

[27] H. Holden and N. H. Risebro. Front tracking for conservation laws. Book in preparation.

[28] K. H. Karlsen, K. Brusdal, H. K. Dahle, S. Evje, and K.-A. Lie. The corrected operator splitting approach applied to a nonlinear advection-diffusion problem. Preprint, University of Bergen, Norway, 1996.

[29] K. H. Karlsen, K.-A. Lie, N. H. Risebro, and J. Frøyen. A front tracking approach to a two-phase fluid flow model with capillary forces. Preprint, University of Bergen, Norway, 1997. Available at the URL http: //www . math.ntnu.no/conservation/.

[30] K. H. Karlsen and N. H. Risebro. Corrected operator splitting for nonlinear parabolic equations. Preprint, University of Bergen, Norway, 1997. Available at the URL http: //www . math.ntnu.no/conservation/.

[31] K. H. Karlsen and N. H. Risebro. An operator splitting method for nonlinear convection-diffusion equations. To appear in Numer. Math. Available at the URL http://www . math.ntnu.no/conservation/.

[32] J. O. Langseth, A. Tveito, and R. Winther. On the convergence of operator splitting applied to conservation laws with source terms. SIAM J. Appl. Math., 33(3):843-863, June 1996.

[33] R. J. LeVeque. Numerical methods for convservation laws. Lectures in Mathematics. ETH Zürich. Birkhäuser Verlag, Basel, second edition, 1992.

[34] K.-A. Lie, V. Haugse, and K. H. Karlsen. Dimensional splitting with front tracking and adaptive grid refinement. Preprint (mathematics). 2, Norwegian University of Science and Technology, 1996. Available at the URL http://www.math.ntnu.no/conservation/.

[35] M. J. Lighthill and G. B. Whitham. On kinematic waves. II. Theory of traffic flow on long crowded roads. Proc. Roy. Soc, 229A:317-345, 1955.

[36] M. S. Mock. Analysis of mathematical models of semi-conductor devices. Boole Press, Dublin, 1983.

[37] K. K. Mohanty, J. M. Ottino, and H. T. Davis. Reaction and transport in disordered composite media. Chem. Eng. Sci., 37:905-924, 1982.

[38] O. A. Oleinik. Discontinuous solutions of non-linear differential equations. Amer. Math. Soc Transl. Ser., 26:95-172, 1963.

[39] N. H. Risebro. A front-tracking alternative to the random choice method. Proc. Amer. Math. Soc, 117(4):1125-1129, Apr. 1993.

[40] T. F. Russell. Galerkin time stepping along characteristics for Burgers' equation. In Stepleman et al., editor, Scientific computing, pages 183-192. IMACS, North-Holland, 1983.

[41] T. F. Russell and R. V. Trujillo. Eulerian-Lagrangian localized adjoint methods with variable coefficients in multiple dimensions. In G. Gambolati et al., editor, Proc. 8th Int. Conf. on Computational Methods in Water Resources, pages 357-363. Computational Mechanics Publications, Southampton, UK, 1990.

[42] J. A. Trangenstein. Mathematical structure of the black-oil model for petroleum reservoir simulation. SIAM J. Appl. Math., 49:749-783, 1989.

[43] A. I. Volpert. The spaces BV and quasi-linear equations. Math. USSR Sbornik, 2(2):225-267, 1967.

[44] H. Wang, H. K. Dahle, M. S. Espedal, R. E. Ewing, R. C. Sharpley, and S. Man. An ELLAM scheme for advection-dispersion equations in two dimensions. Preprint. 
[45] H. Wang and R. E. Ewing. Optimal-order convergence rates for ELLAM for reactive transport and contamination in groundwater. Numerical Methods for Partial Differential Equations, 11:1-35, 1995.

[46] H. Wang, R. E. Ewing, and M. A. Celia. Eulerian-Lagrangian localized adjoint methods for reactive transport with biodegradation. Numerical Methods for Partial Differential Equations, 11:229-254, 1995.

[47] H. Wang, R. E. Ewing, and T. F. Russell. Eulerian-Lagrangian localized adjoint methods and their convergence analysis. IMA J. Numer. Anal., 15, 1995.

[48] M. F. Wheeler, W. A. Kinton, and C. N. Dawson. Time-splitting for advection-dominated parabolic problems in one space variable. Comm. Appl. Numer. Methods., 4:413-423, 1988.

(K. Hvistendahl Karlsen)

DEPARTMENT OF MATHEMATiCS

UNIVERSITY OF BERGEN

Johs. BRunsgt. 12

N-5008 Bergen, Norway

E-mail address: kennethk@mi.uib.no

(K.-A. Lie)

Department of Mathematical Sciences

NorWEgian University OF SCIENCE AND TEChNOLOGY

N-7034 Trondheim, Norway

E-mail address: andreas@math.ntnu.no 